TRANSACTIONS OF THE

AMERICAN MATHEMATICAL SOCIETY

Volume 259, Number 1, May 1980

\title{
EQUIVARIANT DYNAMICAL SYSTEMS
}

BY

M. J. FIELD

\begin{abstract}
The basic properties of vector fields and diffeomorphisms invariant under the action of a compact Lie group are presented. A Kupka-Smale density theorem for equivariant dynamical systems and an existence theorem for equivariant Morse-Smale systems on an arbitrary compact $G$-manifold are proved.
\end{abstract}

Introduction. In this work we develop the basic properties of vector fields and diffeomorphisms invariant under the action of a compact Lie group. Earlier versions of this theory were announced in [7], [8] though these results are weaker in certain significant respects than those presented here.

We prove two fundamental theorems: A Kupka-Smale density theorem for equivariant dynamical systems (Theorem B of \$9) and an existence theorem for equivariant Morse-Smale vector fields on a compact $G$-manifold (Theorem D of $\S 10)$. The latter theorem has interesting implications for the theories of equivariant dynamical systems and equivariant differential topology, notably for a generalisation of the $C^{0}$ isotopy and approximation theorems of Shub and Smale [23], [27] to equivariant maps and we intend to pursue these matters elsewhere. We hope also to make applications of part of our theory to bifurcation problems involving a loss of symmetry (cf. S. Schecter, Bifurcations with symmetry [17, pp. 224-249]). The definition of $\Omega_{G}$ in $\$ 10$ is particularly relevant here as is the theory of equivariant general position or $G$-transversality for the invariant manifolds of elementary critical elements (see $\$ 9$ and also below).

In $\S \S 1,2$ we outline the basic properties of smooth $G$-manifolds and equivariant flows and diffeomorphisms that we need. In $\$ 3$ we define elementary invariant $G$-orbits in terms of the normal hyperbolicity conditions of Hirsh, Pugh and Shub [15] and then give a spectral characterisation of such orbits. In $\$ 4$ we make a similar discussion of periodic orbits followed in $\$ \S 5,6$ by the basic local theory of equivariant dynamical systems. In $\S 7$, we prove that $C^{r}$ 1-generic (resp. 2-generic) equivariant vector fields and diffeomorphisms form an open dense set (resp. residual set), $r \geqslant 1$. Apart from the complications introduced by the presence of the $G$-action, the proofs follow Peixoto [20]. Stable manifold theory is discussed in §8. In \$9, we prove a Kupka-Smale density theorem for $C^{\infty}$ equivariant dynamical systems. As there is yet no $C^{r}$-theory of equivariant general position, $r<\infty$, we have no $C^{r}$ density theorem. It should be pointed out that the theory of equivariant

Received by the editors March 8, 1979.

AMS (MOS) subject classifications (1970). Primary 58F10; Secondary 57E15.

Key words and phrases. Compact Lie group, normal hyperbolicity, equivariant vector field, equivariant diffeomorphism, genericity, equivariant general position. 
general position or $G$-transversality developed independently by $\mathrm{E}$. Bierstone and the author [4], [5], [10] only enters our theory at this point. We do not and cannot, for example, formulate satisfactory nondegeneracy conditions on invariant $G$-orbits in terms of $G$-transversality to the diagonal or zero section of the tangent bundle (cf. Abraham and Robbin [1]). Finally, in $\S 10$, we define the equivariant $\Omega$-set [12] and prove the existence of equivariant Morse-Smale vector fields. Added in proof. An alternative definition of equivariant Morse-Smale vector field has been given by G. L. dos Reis, Structural stability of equivariant vector fields, An. Acad. Brasil Ci. 50 (1978), 273-276.

In their paper on Axiom A actions (Invent. Math. 29 (1975), 7-38), Pugh and Shub prove sufficient conditions for the $\Omega$-stability of a class of actions which includes diffeomorphisms and flows invariant by a compact Lie group. Their results imply, for example, that a (strongly) equivariant Morse-Smale vector field or diffeomorphism is $\Omega$-stable. C. Camacho has also made a study of stability properties of $\mathbf{R}^{k} \times \mathbf{Z}^{l}$ actions (for example: on $\mathbf{R}^{k} \times \mathbf{Z}^{l}$ actions; Morse-Smale $\mathbf{R}^{2}$-actions on two-manifolds, Dynamical systems, Ed. M. M. Peixoto, Academic Press, New York, 1973, pp. 23-70; 71-74). His definition of Morse-Smale for $\mathbf{R}^{2}$-actions may bear some relation to our concept of $\Omega_{G}$ and equivariant MorseSmale. I would like to thank the referee for drawing my attention to the aforementioned works of Pugh, Shub and Camacho.

1. Generalities on $G$-actions. We start by recalling some facts about smooth (that is $C^{\infty}$ ) actions of a compact Lie group on a connected differential manifold. We refer to Bredon's text [6], especially Chapters 5 and 6, for further details and proofs.

Given a connected differential manifold $M$ and the action of a compact Lie group $G$ on $M$, we let $G(x)$ denote the $G$ orbit through $x, G_{x}$ the isotropy subgroup of $G$ at $x$ and $N\left(G_{x}\right)$ the normaliser of $G_{x}$ in $G$. We say $x, y \in M$ are of the same orbit type if $G_{x}$ and $G_{y}$ are conjugate subgroups of $G$ or, equivalently, if $G(x)$ and $G(y)$ are equivariantly diffeomorphic. Equality of orbit types partitions $M$ into points of the same orbit type. If $M$ is compact this partition is finite. We write $M=\cup_{i \in I} M_{i}$ where the $M_{i}$ are the equivalence classes of points of the same orbit type. We define orb: $M \rightarrow I$ by $\operatorname{orb}(x)=i, x \in M_{i}$. There is defined a natural partial order on $I$ by $i<j$ if there exists $x \in M_{i}, y \in M_{j}$ such that $G_{x} \supset G_{y}$ (strict inclusion). $x$ is of minimal orbit type if there does not exist $y \in M$ such that $\operatorname{orb}(y)<\operatorname{orb}(x)$. There exists at least one minimal orbit type. We similarly define maximal orbit type and it may be shown that there exists a unique maximal orbit type, $M_{N}$ say, and that $M_{N}$ is open and dense in $M$ and even connected if $G$ is connected [6, p. 179]. For convenience we label orbit types by positive integers and write $M=\cup_{1<i<N} M_{i}$, where $\operatorname{orb}(x)<\operatorname{orb}(y)$ implies that if $x \in M_{i}$ and $y \in M_{j}$ then $i<j$. We say that the action is principal if there exists only one orbit type and free if $G_{x}=\{e\}$ for all $x \in M$.

If $M$ and $N$ are $G$-manifolds, we let $C_{G}^{r}(M, N)$ denote the space of $C^{r}$ equivariant maps from $M$ to $N$ and $\operatorname{Diff}_{G}^{r}(M)$ denote the group of $C^{r}$ equivariant diffeomorphisms of $M$. We remark that if $M$ is compact and $0<r<\infty$, then 
$C_{G}^{r}(M, N)$ may be given the structure of a Banach manifold [7]. The proof is an equivariant version of that given in Palais [18] for $C^{r}(M, N)$ and uses $\mathrm{A}$. Wassermann's approximation theorem [28, p. 132].

In the sequel, we shall only specify the differentiability class of a map, bundle, submanifold, etc., when it is not $C^{\infty}$. Thus, reference to an "equivariant diffeomorphism of $M$ " will be to an element of $\operatorname{Diff}_{G}^{\infty}(M)$.

The tangent bundle of $M, T M$, has the natural structure of a $G$-vector bundle [6, p. 303] if we define $g(v)=T g(v)$, for $v \in T M, g \in G$.

Averaging a Riemannian metric for $M$ over $G$, we may suppose that $M$ has an equivariant Riemannian metric and corresponding structure of a Riemannian $G$-manifold. The normal bundle of an invariant (that is, $G$-invariant) closed $C^{r}$ submanifold $X$ of $M$ may therefore be given the structure of a $C^{r-1} G$-vector subbundle of $T_{X} M$. Using the equivariance of the exponential map, we may construct an invariant tubular neighbourhood of $X$ [6, pp. 305-307]. In particular, if $X$ is a $G$-orbit $\alpha$, we have the differentiable slice theorem: Given an open neighbourhood $W$ of $\alpha$ and $x \in \alpha$, there exists an equivariant embedding $q$ of $G \times{ }_{H} V$ onto an open invariant tubular neighbourhood $U \subset W$ of $\alpha$, where $H=G_{x}, V$ is the fibre of the normal bundle to $\alpha$ at $x$ and $q(e, 0)=x$. If we set $S_{x}=q(\{e\} \times V), S_{x}$ is a slice for the action of $G$ at $x$.

Continuing with the notation of the differentiable slice theorem, the $G$-vector bundle $G \times{ }_{H} V$ has, by restriction, the structure of an $H$-vector bundle over $\alpha$. We say that $(A \times V, \phi, q)$ is an equivariant chart for $M$ at $x$ if $A$ is an open neighbourhood of $x$ in $\alpha, \phi: A \times V \rightarrow G \times{ }_{H} V$ is an $H$-equivariant trivialisation of $G \times{ }_{H} V$ over $A$, and $q: G \times{ }_{H} V \rightarrow M$ is the tubular map of the differentiable slice theorem. We of ten denote equivariant charts in abbreviated form by $(A \times$ $V, \gamma)$, where it is understood that $\gamma$ is the composite of the trivialisation $\phi$ and tubular map $q$. To construct an equivariant chart for $M$ at $x$, it is sufficient to choose an $H$-invariant open neighbourhood $A$ of $x$ in $\alpha$ and $H$-equivariant local section $p: A \rightarrow G$ satisfying $p(x)=e . \phi: A \times V \rightarrow G \times{ }_{H} V$ is then defined by mapping $(g[H], v)$ to $(p(g[H]), v)$.

If $E$ is a $C^{r} G$-vector bundle over $M$, we let $C_{G}^{s}(E)$ denote the space of $C^{s}$ equivariant sections of $E, 0 \leqslant s \leqslant r$. In case $M$ is compact, we give $C_{G}^{s}(E)$ the $C^{s}$ topology, $s \leqslant \infty\left[14\right.$, p. 34]. Averaging over $G$, it is clear that $C_{G}^{s}(E)$ is a splitting subspace of $C^{s}(E)$.

We shall have occasion to use the following elementary lemmas whose proofs we omit.

Lemma A. Let $E \stackrel{p}{\rightarrow} M$ be a $C^{r} G$-vector bundle. Let $x \in M, U$ be an invariant neighbourhood of $x, Z \in E_{x}$ and $G_{Z}=G_{x}$. Then there exists $s \in C_{G}^{r}(E)$ such that $s(x)=Z$ and $s$ is zero outside $U$.

LEMMA B. For all $x \in M$,

$$
\begin{gathered}
\left\{Y \in T_{x}(G(x)): G_{Y}=G_{x}\right\}=T_{x}\left(N\left(G_{x}\right)(x)\right), \\
\left\{Y \in T_{x} M: G_{Y}=G_{x}\right\}=T_{x}\left(M^{G_{x}}\right),
\end{gathered}
$$

where $M^{G_{x}}$ denotes the fixed set of the action of $G_{x}$ on $M$. 
2. Elementary properties of equivariant flows. If $X$ is a $C^{1}$ vector field on compact $M$, we denote the corresponding flow by $F^{X}: M \times \mathbf{R} \rightarrow M$. In the sequel we often drop the superscript $X$ when no confusion will result. We recall that if $X$ is $C^{r}$ then $F$ is $C^{r}$ and even $C^{r+1}$ in the $\mathbf{R}$ variable. Given $x \in M, t \in \mathbf{R}$, we let $F_{x}: \mathbf{R} \rightarrow M$ and $F_{t}: M \rightarrow M$ denote the corresponding restrictions of $F$. The proof of the first part of the next proposition is a consequence of the uniqueness of integral curves of a vector field.

Proposition A. Let $M$ be a compact $G$-manifold and $X \in C_{G}^{r}(T M), r>1$. Then 1. $F^{X}$ is equivariant: $F^{X}(g x, t)=g F^{X}(x, t), x \in M, t \in \mathbf{R}, g \in G$.

2. The isotropy subgroups of $G$ are constant on integral curves of $F^{X}$.

If $X$ is an equivariant vector field on $M$ and $X(x)=0$ then $X(g x)=0, g \in G$, and so the $G$ orbit through $x$ is singular for $X$. Using 1 of Proposition A, we see that if $\gamma$ is a periodic orbit of $X$ then so is $g(\gamma), g \in G$, and $g(\gamma)$ and $\gamma$ have the same period. We note that since $F_{X}$ is $C^{r+1}$ in the R-variable, $\gamma$ and $G(\gamma)$ are embedded $C^{r+1}$ submanifolds of $M$.

Suppose that $M$ is the homogeneous space $G / H$, where $H$ is a closed subgroup of $G$ and $G$ acts on $G / H$ by left translation. Since $G$ acts transitively, an equivariant vector field on $G / H$ is determined by its value on the identity coset $[H]$ and is necessarily $C^{\infty}$. Moreover, if $\mathfrak{n}$ and $\mathfrak{h}$ denote the Lie algebras of $N(H)$ and $H$ respectively, Lemma B of $\S 1$ implies $C_{G}^{\infty}(T(G / H)) \simeq \mathfrak{n} / \mathfrak{h}$. We set $R(G / H)$ $=\operatorname{rank}(N(H) / H)$.

Proposition B1. Let $X \in C_{G}^{\infty}(T(G / H))$. Then there exists an $X$-invariant foliation $\mathscr{F}_{X}=\left\{T_{x}^{s}: x \in G / H\right\}$ of $G / H$ by s-dimensional tori such that

$1.0<s \leqslant R(G / H)$.

2. $T_{x}^{s}$ is the closure of the $X$-orbit through $x, x \in G / H$.

3. $F^{X} \mid T_{x}^{s}$ is zero, a periodic orbit or an irrational torus flow depending on whether $s=0, s=1$, or $s>1$ respectively.

Proof. Let $x=[H]$ and $O(x)$ denote the orbit of $X$ through $x$. Now $\overline{O(x)} \subset$ $N(H) / H$ and clearly $\overline{O(x)}$ has the structure of a compact connected abelian subgroup of $N(H) / H$. Hence, $\overline{O(x)}$ is an $s$-dimensional torus, $s<R(G / H)$. The statements of the proposition follow.

Remark. Given $X \in C_{G}^{\infty}(T(G / H))$, we can always find $X^{\prime}$, arbitrarily close to $X$ in the $C^{\infty}$ topology such that the foliation corresponding to $X^{\prime}$ given by Proposition B1 is by $R(G / H)$-dimensional tori.

Suppose that $G$ acts principally on $M$ and the orbit space of $M$ is diffeomorphic to $S^{1}$. The fibres of the orbit map $\pi: M \rightarrow S^{1}$ are equivariantly diffeomorphic to the homogeneous space $G / H$, where $H$ is any isotropy subgroup of the action of $G$ on $M . \pi$ induces a surjection $\pi_{*}: C_{G}^{r}(T M) \rightarrow C^{r}\left(T S^{1}\right), r>1$, which is injective if and only if $R(G / H)=0$. Indeed, this is an immediate consequence of Lemma $B$ of $\$ 1$ since $\operatorname{Ker} \pi_{*}=\left\{X \in C_{G}^{r}(T M): X(x) \in T_{x} G(x), x \in M\right\}$. Let $C^{r}\left(T S^{1}\right)^{*}$ denote the set of nowhere vanishing $C^{r}$ vector fields on $S^{1}$. If $X \in C^{r}\left(T S^{1}\right)^{*}$, the 
flow of $X$ consists of a single limit cycle. If $N(H) / H$ is finite, the flow of $\pi_{*}^{-1}(X)$ will consist of periodic orbits. More generally, we have

Proposition B2. Let $X \in \pi_{*}^{-1}\left(C^{r}\left(T S^{1}\right)^{*}\right)$. Then there exists a $C^{r+1}$ foliation $\mathscr{F}_{X}=\left\{T_{x}^{p}: x \in M\right\}$ of $M$ by $p$-dimensional $X$-invariant tori, where

$1.0<p \leqslant R(G / H)+1$.

2. $T_{x}^{p}$ is the closure of the $X$-orbit through $x, x \in M$.

3. $F^{X} \mid T_{x}^{p}$ is a periodic orbit or irrational torus flow according to whether $p=0$ or $p>0$ respectively.

Proof. Fix $x \in M$ and set $G_{x}=H, M_{x}=\pi^{-1}(\pi x)$ and $M^{H}=\left\{y \in M: G_{y}=\right.$ $H$ \}. The orbit of $x, O(x)$, is contained in the $N(H)$-space $M^{H}$ and $M^{H} \cap M_{x}=$ $N(H) / H$, where the inclusion of $N(H) / H$ in $G / H=M_{x}$ is induced by that of $N(H)$ in $G$. We regard $x=[H]$ as being the identity element of the group $N(H) / H \subset M_{x}$. Let $T$ be the smallest strictly positive real number such that $F(x, T) \in M_{x}\left(T\right.$ is the period of the orbit of $\pi_{*} X$ and is independent of $\left.x\right)$. There exists a unique $h \in N(H) / H$ such that $F(x, T)=h$ and clearly $O(x) \cap M_{x}=$ $\left\{h^{n}: n \in \mathbf{Z}\right\}=\Gamma$, say. We let $\bar{\Gamma}$ denote the closure of $\Gamma$ in $N(H) / H$. $\bar{\Gamma}$ is a compact abelian subgroup of $N(H) / H$. $h$ induces an equivariant map $\tilde{h}: \bar{\Gamma} \rightarrow \bar{\Gamma}$ by $g \mapsto g h$. We now suspend $\tilde{h}$ as in Smale [26, p. 797] and consider the manifold $K=(\bar{\Gamma} \times \mathbf{R}) / \sim$, where $\sim$ is defined by $(g, t+T) \sim(g h, t), g \in \bar{\Gamma}, t \in \mathbf{R}$. The product abelian Lie group structure on $\bar{\Gamma} \times \mathbf{R}$ drops down to an abelian Lie group structure on $K$. But $K$ is $C^{r+1}$ diffeomorphic to $\overline{O(x)}$ by the map induced from $(g, t) \mapsto F(g x, t)$. In particular, $K$ is connected and therefore a torus. The dimension of $K$ is equal to $1+\operatorname{dimension}(\bar{\Gamma})<1+R(G / H)$. Translating $\overline{O(x)}$ in $M$ using $G$, we obtain the remaining statements of the proposition.

Remarks. As in Proposition B1, given $X \in \pi_{*}^{-1}\left(C^{r}\left(T S^{1}\right)\right)^{*}$, we can always $C^{r}$ approximate $X$ by $X^{\prime}$ so that the toral foliation corresponding to $X^{\prime}$ is of maximal dimension. Proposition B2 can clearly be generalised to principal $G$-manifolds, orbit space a torus and equivariant flow covering an irrational torus flow.

3. Generic critical elements of equivariant vector fields and diffeomorphisms. We start by giving a technical definition which guarantees the stability of flow invariant $G$-orbits within points of fixed orbit type. Suppose that $i$ is an orbit type and let $T_{i}(G) \subset T\left(M_{i}\right)$ denote the $G$-vector subbundle of tangents to $G$-orbits. That is,

$$
T_{i}(G)=\bigcup_{x \in M_{i}} T_{x}(G(x))
$$

Definition Al. If $\alpha$ is a $G$-orbit of type $i$ which is invariant by the flow of $X \in C_{G}^{1}(T M)$, we shall say that $\alpha$ is nondegenerate for $X$ on $M_{i}$ if $X \mid M_{i}$ : $M_{i} \rightarrow T\left(M_{i}\right)$ is transversal to $T_{i}(G)$ along $\alpha$. We say $X$ is 0 -generic on $M_{i}$ if every $X$-invariant $G$-orbit in $M_{i}$ is nondegenerate for $X$ on $M_{i}$.

We note that if $\alpha$ is nondegenerate for $X$ on $M_{i}$ then $\alpha$ is certainly an isolated $X$-invariant $G$-orbit in $M_{i}$. However, it need not be isolated in $M$. For example, if 
we take $X$ identically zero on $\mathrm{C}$ and the standard representation of $S^{1}$ on $\mathrm{C}$, then the origin is nondegenerate for $X$ on the fixed point set of $S^{1}$ but it is certainly not isolated in the singular set of $X$ on $\mathbf{C}$.

Suppose $\alpha$ is an $X$-invariant $G$-orbit and $(A \times V, \gamma)$ is an equivariant chart for $M_{i}$ at $x \in \alpha$ (see $\S 1$-note $G_{x}$ acts trivially on $V$ ). Let $q: V \rightarrow A \times V$ denote the inclusion of $V$ as $\{x\} \times V$ and $p$ denote the projection of $A \times V$ on $V$. Set $X_{\gamma}^{i}=T p(q \gamma)^{*} X \in C^{1}(T V)$. The following trivial lemma gives a local description of nondegenerate $G$-orbits.

Lemma B1. Let $X \in C_{G}^{1}(T M)$ and $\alpha \subset M_{i}$ be an $X$-invariant $G$-orbit. $\alpha$ is nondegenerate for $X$ on $M_{i}$ if and only if given $x \in \alpha$ and any equivariant chart $(A \times V, \gamma)$ for $M_{i}$ at $x, X_{\gamma}^{i}$ is transverse to the zero section of TV at $x$. Furthermore, if $X_{\gamma}^{i}$ is transverse to the zero section of $T V$ on $V$, then every $X$-invariant $G$-orbit in $G(\gamma(A \times V)) \subset M_{i}$ is nondegenerate.

Turning now to $G$-orbits invariant by an equivariant diffeomorphism, we let $\Delta_{i}(G)=\left\{(x, g(x)): x \in M_{i}, g \in G\right\} . \Delta_{i}(G)$ is an invariant submanifold of $M_{i} \times$ $M_{i}$.

Definition A2. If $\alpha$ is a $G$-orbit of type $i$ which is $f$-invariant, $f \in \operatorname{Diff}_{G}^{1}(M)$, we say $\alpha$ is nondegenerate for $f$ on $M_{i}$ if $\operatorname{graph}(f) \mid M_{i}: M_{i} \rightarrow M_{i} \times M_{i}$ is transverse to $\Delta_{i}(G)$ along $\alpha$. We say $f$ is 0 -generic on $M_{i}$ if every $f$-invariant $G$-orbit in $M_{i}$ is nondegenerate for $X$ on $M_{i}$.

As in the case of flows we may give a simple characterisation of nondegenerate orbits for $f$ on $M_{i}$. Thus, if $\alpha$ is $f$-invariant and $x \in \alpha$ there exists $g \in N\left(G_{x}\right)$ such that $g f(x)=x$. Let $(A \times V, \gamma)$ be an equivariant chart for $M_{i}$ at $x$ and $q$ and $p$ be as defined prior to Lemma B1. We let $f_{\gamma}^{i}: V \rightarrow V$ be the map $p \gamma^{-1} g f \gamma$, which is certainly defined for small values of $v \in V$.

Lemma B2. Let $f \in \operatorname{Diff}_{G}^{1}(M)$ and $\alpha \subset M_{i}$ be an $f$-invariant G-orbit. $\alpha$ is nondegenerate for $f$ on $M_{i}$ if and only if given $x \in \alpha, g \in N\left(G_{x}\right)$ such that $g f(x)=x$, any equivariant chart $(A \times V, \gamma)$ for $M_{i}$ at $x$, then 1 does not belong to the spectrum of $D f_{\gamma}^{i}(0)$.

Proof. Similar to that of Lemma B1. Both results follow from more general theory given below.

Our intention now is to frame a definition of hyperbolicity suitable for the study of invariant $G$-orbits of an equivariant flow or diffeomorphism. We shall formulate our definitions in terms of the normal hyperbolicity conditions of Hirsch, Pugh and Shub [15]. Suppose $V$ is a closed $C^{1}$ submanifold of $M$. For our purposes the following definition of normal hyperbolicity at $V$ ("Immediately, absolutely 1-normally hyperbolic" in [15]) will suffice.

$V$ is normally hyperbolic for $f \in \operatorname{Diff}^{1}(M)$ if $V$ is $f$-invariant and there exists a $T f$-invariant splitting $T V \oplus N^{u} \oplus N^{s}$ of $T_{V} M$ into continuous subbundles such that, relative to some Riemannian metric on $M$, 


$$
\begin{aligned}
& \sup _{x \in V}\left\|T f \mid N_{x}^{s}\right\|<\inf _{x \in V} m\left(T f \mid T_{x} V\right), \\
& \sup _{x \in V}\left\|T f \mid T_{x} V\right\|<\inf _{x \in V} m\left(T f \mid N_{x}^{u}\right), \\
& \quad\left(m(A)=\inf \{\|A X\|:\|X\|=1\}=\left\|A^{-1}\right\|^{-1}\right) .
\end{aligned}
$$

We shall always assume that $V$ is $G$-invariant and $f \in \operatorname{Diff}_{G}^{1}(M)$. It is then easy to show that the Riemannian metric of the definition may be taken to be equivariant and that the bundles $N^{u}$ and $N^{s}$ are $C^{0} G$-vector bundles over $V$.

If $F^{X}$ is a $C^{1}$ equivariant flow on $M$ leaving $V$ invariant, $F^{X}$ is said to be normally hyperbolic at $V$ if, for some $s \neq 0, F_{s}^{X}$ is normally hyperbolic at $V$. By Theorem 2.4 of [15], $F_{t}^{X}$ is then normally hyperbolic at $V$ for all $t \neq 0$ and the corresponding splittings of $T_{V} M$ are independent of $t$.

Definition C1. Let $\alpha$ be a $G$-orbit which is invariant by the flow of $X \in$ $C_{G}^{1}(T M)$. We say that $\alpha$ is an elementary or generic $G$-orbit for $X$ if $F^{X}$ is normally hyperbolic at $\alpha$.

Definition C2. If $\alpha$ is a $G$-orbit which is $f$-invariant, $f \in \operatorname{Diff}_{G}^{1}(M)$, we say $\alpha$ is an elementary or generic $G$-orbit for $f$ if $f$ is normally hyperbolic at $\alpha$.

REMARK. Since $G$ acts transitively on a $G$-orbit $\alpha$, the splittings $T \alpha \oplus N^{u} \oplus N^{s}$ corresponding to normal hyperbolicity at $\alpha$ are always $C^{\infty}$.

ExAmples. Let $X \in C_{G}^{1}(T M)$ and suppose that $\alpha$ is an $X$-invariant $G$-orbit and $X$ is zero on $\alpha$. Let dimension $(\alpha)=p$, dimension $(M)=m$. At every point $x \in \alpha$, we may define the Hessian of $X, \dot{X}(x) \in L\left(T_{x} M, T_{x} M\right)[1$, p. 59]. $\dot{X}(x)$ has at least $p$ zero eigenvalues corresponding to the $\dot{X}(x)$-invariant subspace $T_{x} \alpha$ of $T_{x} M$. We claim that $\alpha$ is an elementary $G$-orbit for $X$ if and only if $\dot{X}(x)$ has $m-p$ eigenvalues with nonzero real part. Indeed, suppose $\dot{X}(x)$ has $m-p$ eigenvalues with nonzero real part, then $T_{x} M$ has an $\dot{X}(x)$-invariant splitting $T_{x} \alpha \oplus N_{x}^{u} \oplus N_{x}^{s}$, where $N_{x}^{u}$ and $N_{x}^{s}$ are the subspaces of $T_{x} M$ corresponding to eigenvalues of $\dot{X}(x)$ with positive and negative real part respectively. Since $\dot{X}(g x)=g \dot{X}(x) g^{-1}$, we have a $C^{\infty}$ splitting $T \alpha \oplus N^{u} \oplus N^{s}$ of $T_{\alpha} M$ into $G$ vector subbundles and this splitting is obviously $T F_{t}^{X}$-invariant. Observing that for all $x \in \alpha,\left\|T_{x} F_{t} \mid T_{x} \alpha\right\|=1$, the normal hyperbolicity of $F^{X}$ at $\alpha$ follows. The converse is similarly proved.

Let $f \in \operatorname{Diff}_{G}^{1}(M)$ and $\alpha$ be an $f$-invariant $G$-orbit such that $f$ restricts to the identity on $\alpha$. Then $\alpha$ is an elementary $G$-orbit for $f$ if and only if given $x \in \alpha$, $T_{x} f \in L\left(T_{x} M, T_{x} M\right)$ has $m-p$ eigenvalues of modulus not equal to one. The proof is similar to that given above for flows.

Motivated by the above examples our aim is now to give spectral conditions that will guarantee that an invariant $G$-orbit is elementary. We first recall some elementary representation theory of compact Lie groups. (Our terminology follows that of Adams [3].)

If the vector space $V$ is a (real) $H$-representation, we let $L_{H}(V, V)$ denote the set of $H$-equivariant linear endomorphisms of $V$. We recall Frobenius' theorem [21, p. 160].

LEMMA D. Let $V$ be a real irreducible representation of $H$. Then

$$
L_{H}(V, V) \cong \mathbf{F} \text { (as algebras), }
$$

where $\mathbf{F}=\mathbf{R}, \mathbf{C}$ or $\mathbf{H}$. 
(For our applications we shall only be interested in the case where $H$ is compact abelian and then $\mathbf{F}=\mathbf{R}$ or $\mathbf{C}$.)

Definition. Let $V$ be a real vector space and $A \in L(V, V)$. We define the reduced spectrum of $A, \operatorname{spec}(A)$, to be $\operatorname{spectrum}(A) / S^{1} \subset \mathrm{C} / S^{1}$. We similarly define $\operatorname{spec}(M)$, for $M$ a square matrix (coefficients in $\mathbf{R}, \mathbf{C}$ or $\mathbf{H}$ ).

RemarKs. In the definition, $S^{1}$ acts on $\mathrm{C}$ multiplicatively and $\mathrm{C} / S^{1} \simeq \mathbf{R}(>0)$. In the sequel we often $\operatorname{regard} \operatorname{spec}(A) \subset \mathbf{R}(>0)$.

Given a real $H$-representation $V, V \cong \bigoplus_{i=1}^{p} V_{i}^{r_{i}}$, where the $V_{i}$ are mutually nonisomorphic R-irreducible representations of $H$ and the superscript $r_{i}$ denotes $r_{i}$-fold direct sum. Let $\mathrm{F}_{i}=L_{H}\left(V_{i}, V_{i}\right)$ and $M\left(r_{i}, \mathrm{~F}_{i}\right)$ denote the ring of $r_{i} \times r_{i}$ matrices with coefficients in $F_{i}$. By Schur's lemma and Lemma $D$, there exists a real isomorphism

$$
\chi: L_{H}(V, V) \rightarrow \bigoplus_{i=1}^{p} M\left(r_{i}, F_{i}\right)
$$

and from Lemma $\mathrm{D}$ it follows that $\operatorname{spec}(\chi(A))=\operatorname{spec}(A), A \in L_{H}(V, V)$.

Remark. If all the $\mathbf{F}_{i}=\mathbf{R}$ then, of course, $\operatorname{spectrum}(\chi(A))=\operatorname{spectrum}(A)$. If $\mathbf{F}_{i}=\mathbf{R}$ or $\mathbf{C}$, then $\operatorname{spectrum}(A) /( \pm 1)=\operatorname{spectrum}(\chi(A)) /( \pm 1)$.

LEMMA E. Let $H$ be a compact abelian Lie group and $V$ be a real representation of H. Then

$$
\operatorname{spec}(h A)=\operatorname{spec}(A), \quad A \in L_{H}(V, V), h \in H
$$

Proof. Since $H$ is abelian, any $h \in H$ is an $H$-equivariant endomorphism of $V$. The lemma follows easily using the above structure theory for $L_{H}(V, V)$ together with the fact that the action of $H$ on an irreducible representation is just scalar multiplication by real or complex numbers of absolute value one.

Proposition F. Let $H$ be a compact subgroup of the compact abelian Lie group $K$. Suppose $E$ is a $K$-vector bundle over $K / H$ and that $A: E \rightarrow E$ is a $K$-vector bundle map covering the $K$-equivariant diffeomorphism $a: K / H \rightarrow K / H$. For $x \in K / H$, choose $k \in K$ such that $k a(x)=x$ and $\operatorname{set} \operatorname{spec}(A ; x, k)=\operatorname{spec}\left(k A: E_{x} \rightarrow E_{x}\right)$. Then $\operatorname{spec}(A ; x, k)$ is independent of the choice of $x$ and $k$ and depends only on $A$.

Proof. Since $K$ is abelian, $K_{x}=H$ for all $x \in K / H$ and so we have a real representation of $H$ on $E_{x}$ for all $x \in K / H$. Also, if $k a(x)=x$ for some $x \in$ $K / H$, then $k a(y)=y$ for all $y \in K / H$ and it follows easily that it is enough to prove the independence of $\operatorname{spec}(A ; x, k)$ from $k$. But if $k a(x)=k^{\prime} a(x), k^{\prime} A=$ $\left(k^{\prime} k^{-1}\right) k A$ and so the result follows from Lemma E, since $k^{\prime} A, k A \in L_{H}\left(E_{x}, E_{x}\right)$.

Theorem G. Let $H$ be a compact subgroup of $G$ and $E$ be $a$-vector bundle over $G / H$. Suppose $A: E \rightarrow E$ is a G-vector bundle map covering the equivariant diffeomorphism $a: G / H \rightarrow G / H$. Given $x \in G / H$, choose $g \in G$ such that $g a(x)=$ $x$ and $\operatorname{set} \operatorname{spec}(A ; x, g)=\operatorname{spec}\left(g A: E_{x} \rightarrow E_{x}\right)$. Then $\operatorname{spec}(A ; x, g)$ is independent of the choice of $x$ and $g$ and depends only on $A$. 
Proof. As in the proof of Proposition $\mathrm{F}$, the problem is to show that $\operatorname{spec}(A$ : $x, g)$ does not depend on $g$. Suppose first that $G$ is finite and that $g a(x)=g^{\prime} a(x)=$ $x, g, g^{\prime} \in G$. Then $g^{n} A^{n}=g^{\prime n} A^{n}, n \geqslant 0$ and choosing $n$ such that $g^{n}=g^{\prime n}=e$, we see that $\operatorname{spec}(g A)^{n}=\operatorname{spec}\left(g^{\prime} A\right)^{n}=\operatorname{spec} A^{n}$ and therefore $\operatorname{spec}(g A)=\operatorname{spec}\left(g^{\prime} A\right)$. To prove the general case, let $x=[H] \in G / H$ and suppose that $N(H)$ is connected. We can assume that $g$ belongs to a maximal torus $T$ of $N(H)$. Suppose $g^{\prime} \in T$ also satisfies $g^{\prime} a(x)=x$. Then $g^{-1} g^{\prime} \in H \cap T=S$ which is compact abelian. But $E_{x}$ is an $S$-representation and so we can apply Proposition $F$ to deduce that $\operatorname{spec}(g A)=\operatorname{spec}\left(g^{\prime} A\right)$. But any $g^{\prime} \in N(H)$ satisfying $g^{\prime} a(x)=x$ is a conjugate of some $g \in T$ satisfying $g a(x)=x$. That is, $g^{\prime}=p^{-1} g p, p \in N(H)$. Since $p^{-1} g p A=p^{-1}(g A) p$, we again see that $\operatorname{spec}\left(g^{\prime} A\right)=\operatorname{spec}(g A)$. If $N(H)$ is not connected, we can always find $m \geqslant 1$ such that $a^{m}(x)$ lies in the orbit of the identity component of $N(H)$. The previous argument then applies together with that given for finite groups.

Definition. Let $E$ be a $G$-vector bundle over $G / H$ and $A$ be a $G$-vector bundle map covering an equivariant diffeomorphism of $G / H$. We define the reduced spectrum of $A, \operatorname{spec}(A)$, to be $\operatorname{spec}(A ; x, g)$ where $x$ and $g$ are chosen as in the statement of Theorem $\mathrm{G}$.

LEMMA H. Let $\alpha$ be an elementary $G$-orbit for $f \in \operatorname{Diff}_{G}^{1}(M)$, and $T \alpha \oplus N^{u} \oplus N^{s}$ denote the corresponding splitting of $T_{\alpha} M$. Then there exists an equivariant Riemannian metric on $M$ such that

1. $\left\|T_{x} f(V)\right\|=\|V\|$, for $V \in T_{x} \alpha, x \in \alpha$.

2. $\sup _{x \in \alpha}\left\|T_{x} f\left|N_{x}^{u}\left\|>1, \inf _{x \in \alpha}\right\| T_{x} f\right| N_{x}^{s}\right\|<1$.

3. The splitting $T \alpha \oplus N^{u} \oplus N^{s}$ is an orthogonal direct sum.

Proof. Fix $x \in \alpha$ and let $G_{x}=H$. Then $\alpha \cong G / H$ with $x=[H]$. We define an action of $G \times N(H)$ on $\alpha$ by

$$
(g, n)(k[H])=g k n^{-1}[H], \quad g \in G, n \in N(H), k[H] \in \alpha .
$$

Since $f$ is a diffeomorphism, $G_{f(x)}=G_{x}=H$. Hence $f(x)=n x$, for some $n \in$ $N(H)$. Consequently, $f(y)=(e, n) y$ for all $y \in \alpha$. Averaging a Riemannian metric for $T \alpha$ over $G \times N(H)$, we see that $T f \mid T \alpha$ will be isometric in the averaged metric. Since a $G \times N(H)$-equivariant metric on $T \alpha$ is necessarily $G$-equivariant, we have therefore found an equivariant metric on Ta satisfying 1. Pick $g \in N(H)$ so that $g f(x)=x$ and choose an inner product (not necessarily $H$-equivariant) on $N_{x}^{u}$ so that $\left\|g T f \mid N_{x}^{u}\right\|<1$. Averaging the inner product over $H$ this condition still holds and so, extending the metric equivariantly over $N^{u}$ and noting that $g^{-1}$ will be an isometry, we obtain 2 for $N^{u}$. Similarly for $N^{s}$. Extend the inner products on $T \alpha$, $N^{u} \oplus N^{s}$ equivariantly to $T_{\alpha} M$ by requiring that the splitting $T \alpha \oplus N^{u} \oplus N^{s}$ is orthogonal. Finally, extend the Riemannian metric on $T_{\alpha} M$ to $T M$ and average over $G$.

Corollary to Lemma $\mathrm{H}$. If $\alpha$ is an $f$-invariant $G$-orbit, $f \in \operatorname{Diff}_{G}^{1}(M)$, then $\operatorname{spec}(T f \mid T \alpha)=1$. 
If $\alpha$ is an $f$-invariant $G$-orbit, $T f: T_{\alpha} M \rightarrow T_{\alpha} M$. Hence $T f$ induces a $G$-vector bundle isomorphism $N_{\alpha} f$ of the normal bundle $N \alpha=T_{\alpha} M / T \alpha$ of $\alpha$.

Theorem I. Let $\alpha$ be an $f$-invariant $G$-orbit, $f \in \operatorname{Diff}_{G}^{1}(M)$. Then $\alpha$ is an elementary $G$-orbit for $f$ if and only if $1 \notin \operatorname{spec}\left(N_{\alpha} f\right)$.

Proof. $\operatorname{Spec}\left(T f \mid T_{\alpha} M\right)=\operatorname{spec}(T f \mid T \alpha) \cup \operatorname{spec}(T f \mid N \alpha)=1 \cup \operatorname{spec}(T f \mid N \alpha)$, Corollary to Lemma $\mathbf{H}$. The result is now immediate from Lemma $\mathbf{H}$.

REMARK. If $f \mid \alpha$ is the identity or $M$ is a principal $G$-manifold then $\operatorname{spec}\left(N_{\alpha} f\right)$ and $\operatorname{spec}\left(T f \mid T_{\alpha} M\right)$ can be defined as subsets of $\mathrm{C}$ rather than just $\mathrm{C} / S^{1}$.

We turn now to the problem of finding a spectral characterisation for elementary $G$-orbits of equivariant vector fields.

Fix $x \in M$ and let $\mathfrak{g}, \mathfrak{h}$ and $\mathfrak{n}(\mathfrak{h})$ denote the Lie algebras of $G, H=G_{x}$ and $N(H)$ respectively. We have a natural map $\theta: g \rightarrow T_{x} M$ defined by $\theta(\zeta)=$ $\left.(d / d t)(\exp (t \zeta)(x))\right|_{t=0}$ (see, for example, [16, p. 42]). Since $\operatorname{Kernel}(\theta)=\mathfrak{h}$ and $\theta(\mathfrak{n}(\mathfrak{h}))=T_{x}(N(H)(x)), \quad \theta$ induces the isomorphism between $\mathfrak{n}(\mathfrak{h}) / \mathfrak{h}$ and $C_{G}^{\infty}(T(G / H))$ referred to in $\S 2$. Let $\tilde{\theta}: \mathfrak{n}(\mathfrak{h}) \rightarrow C^{\infty}(T M)$ denote the map defined by $\tilde{\theta}(\zeta)(y)=\left.(d / d t)(\exp (t \zeta)(y))\right|_{t=0}$.

Proposition J. Let $\alpha$ be an $X$-invariant G-orbit, $X \in C_{G}^{1}(T M)$. Let $x \in \alpha$, $H=G_{x}$ and choose $\zeta \in \mathfrak{n}(\mathfrak{h})$ such that $X(x)=\theta(\zeta)$. Set $\operatorname{Hess}(X, \alpha ; x, \zeta)=$ spectrum of Hessian $(X-\tilde{\theta}(\zeta))(x) / i \mathbf{R}$. Then $\operatorname{Hess}(X, \alpha ; x, \zeta)$ is independent of the choices of $x$ and $\zeta$ and depends only on $X$ and $\alpha$.

Proof. We omit the proof which is similar to that of Theorem G. The quotient $\mathbf{C} / \boldsymbol{i} \mathbf{R}$ is, of course, relative to the additive structure on $\mathbf{C}$.

Definition. Let $\alpha$ be an $X$-invariant $G$-orbit, $X \in C_{G}^{1}(T M)$. We define the reduced Hessian of $X$ on $\alpha, \operatorname{Hess}(X, \alpha)$, to be $\operatorname{Hess}(X, \alpha ; x, \zeta)$, where $x$ and $\zeta$ are chosen as in the statement of Proposition $\mathbf{J}$.

REMARK. If either $X$ is zero on $\alpha$ or $M$ is a principal $G$-manifold, $\operatorname{Hess}(X, \alpha)$ may be defined as a subset of $\mathrm{C}$. If $M=\alpha$ then, as in the proof of Lemma $\mathrm{H}$, it may be shown that $\operatorname{Hess}(X, \alpha)=0$ for all $X \in C_{G}^{\infty}(T \alpha)$.

As a corollary of Proposition $J$ and the above remark we have

Theorem K. An $X$-invariant $G$-orbit $\alpha$ of $X \in C_{G}^{1}(T M)$ is elementary if and only if the multiplicity of 0 in $\operatorname{Hess}(X, \alpha)$ equals the dimension of $\alpha$.

\section{Periodic orbits.}

Definition A. Let $f \in \operatorname{Diff}_{G}^{1}(M)$ and $\alpha$ be a $G$-orbit. $\alpha$ is said to be a $G$-orbit of period $p$ for $f$ if $\alpha$ is invariant by $f^{p}$ and $p$ is the smallest strictly positive integer for which this is true. The set $\left\{\alpha, f(\alpha), \ldots, f^{p-1}(\alpha)\right\}$ is referred to as an $f$-orbit of period $p$.

Definition B. Let $\Lambda$ be an $f$-orbit of period $p, f \in \operatorname{Diff}_{G}^{1}(M) . \Lambda$ is said to be an elementary or generic periodic orbit of $f$ if $f$ is normally hyperbolic at $\Lambda$.

REMARKS. $\Lambda$ is an elementary $f$-orbit of period $p$ if and only if $\Lambda$ consists of $p$ elementary orbits for $f^{p}$. Since the splitting of $T_{\Lambda} M$ for $f^{p}$ is the same as for $f$, we may assume that the splitting is $C^{\infty}$, orthogonal and $T f \mid T \Lambda$ acts as isometries. 
For the remainder of this section, $X$ will always denote an equivariant vector field on $M$ of class at least $C^{1}$.

Let $\Lambda$ be a compact $G$ - and $X$-invariant subset of $M$ and suppose $\Lambda / G \cong S^{1}$. The flow on $\Lambda$ is then described by Proposition B2 of $\S 2$. Let $T$ denote the period of the induced flow on $S^{1}$. T is characterised as being the smallest positive number such that $F(x, T) \in G(x)$ for all $x \in \Lambda$. In the sequel, we shall refer to $\Lambda$ as a periodic $X$-orbit (of period $T$ ). Fix a $G$-orbit $\alpha \subset \Lambda$. Let $N$ denote the restriction of the normal bundle of $\Lambda$ to $\alpha$ and $q: N \rightarrow M$ denote the corresponding restriction of a tubular map to $N . q$ is then a $C^{\infty}$ equivariant embedding (see $\S 1$ and note that although $X$ is only assumed $C^{1}$ the restriction of any $G$-subbundle of $T_{\Lambda} M$ to a $G$-orbit is always $C^{\infty}$ ). Set $D=q(N) . D$ is a $G$-invariant submanifold of $M$ containing $\alpha$ and transverse to $\Lambda$. Since $X$ is tangent to $\Lambda$, we may suppose that $q$ is chosen so that $X$ is transverse to $D$. Suppose $0<a \ll T$. Let $N^{\prime}$ be an open invariant disc bundle of $N$ and set $D^{\prime}=q\left(N^{\prime}\right) \subset D$. By the continuity of $F$, we may choose $N^{\prime}$ of sufficiently small radius such that for each $y \in D^{\prime}$ there exists a unique $\rho(y) \in[T-a, T+a]$ satisfying $F(y, \rho(y)) \in D$. We now define $P: D^{\prime} \rightarrow$ $D ; \rho: D^{\prime} \rightarrow \mathbf{R}$ by $P(y)=F^{X}(y, \rho(y))$. We remark that $\rho(y)$ is the smallest strictly positive number $t$ such that $F^{X}(y, t) \in D$ and that $\rho(x)=T, x \in \alpha$. We call $\left(D, D^{\prime}, P, \rho\right)$ a Poincaré system for $\Lambda$. As for the Poincaré map of a periodic orbit, we may show, using the implicit function theorem and a flow box, that if $X$ is $C^{r}$ then so are $P$ and $\rho$ [2]. $P$ and $\rho$ are also $G$-invariant, where we take the trivial $G$-action on $\mathbf{R}$.

Definition $C$. We say that $\Lambda$ is a generic or elementary periodic $X$-orbit if, for some choice $\left(D, D^{\prime}, P, \rho\right)$ of Poincaré system for $\Lambda, \alpha=D \cap \Lambda$ is an elementary $G$-orbit for $P$.

REMARKS. As in [2], we may easily show that the above definition does not depend on the choice of Poincaré system for $\Lambda$.

Proposition D. $\Lambda$ is an elementary periodic orbit of $X$ if and only if $X$ is normally hyperbolic at $\Lambda$.

Proof. The splitting of $T_{\Lambda} M \mid \alpha, \alpha$ a $G$-orbit in $\Lambda$, coming from the normal hyperbolicity of $P$, extends to a splitting of $T_{\Lambda} M$.

Remarks. If $X$ is of class $C^{r}$, then the bundles $T \Lambda, N^{u}$ and $N^{s}$ are $C^{r} G$-vector bundles since $\Lambda$ is of class $C^{r+1}$. We cannot, however, require the splitting $T \Lambda \oplus N^{u} \oplus N^{s}$ to be orthogonal (unless $X$ is $C^{\infty}$ ) nor can we assume that $T f$ acts as isometries on $T \Lambda$.

5. Local theory of elementary orbits. Most of the results of this section are straightforward applications of the theory presented in [15]. Assertions about equivariance follow by carefully checking that the constructions in the proofs from [15] that we use can all be made equivariant.

TheOReM A. Let $f \in \operatorname{Diff}_{G}^{r}(M)$ and suppose that $\Lambda$ is an elementary periodic orbit for $f$. Then

(a) There exist $C^{r}$, G-invariant, locally f-invariant submanifolds $W_{\text {loc }}^{u}(\Lambda)$ and $W_{\text {loc }}^{s}(\Lambda)$ of $M$ tangent at $\Lambda$ to $T \Lambda \oplus N^{u}$ and $T \Lambda \oplus N^{s}$ respectively. 
(b) Any locally f-invariant set near $\Lambda$ lies in $W_{\text {loc }}^{u}(\Lambda) \cup W_{\text {loc }}^{s}(\Lambda)$.

(c) There exists an invariant neighbourhood $U$ of $\Lambda$ such that

$$
\begin{aligned}
& W_{\text {loc }}^{u}(\Lambda)=\left\{z \in U: f^{n}(z) \in U, n \leqslant 0, \text { and } d\left(f^{n}(z), \Lambda\right) \rightarrow 0 \text { as } n \rightarrow-\infty\right\} . \\
& W_{\text {loc }}^{s}(\Lambda)=\left\{z \in U: f^{n}(z) \in U, n \geqslant 0, \text { and } d\left(f^{n}(z), \Lambda\right) \rightarrow 0 \text { as } n \rightarrow+\infty\right\} .
\end{aligned}
$$

(d) $W_{\text {loc }}^{u}(\Lambda)$ and $W_{\text {loc }}^{s}(\Lambda)$ have the structure of $C^{r}$ equivariant locally trivial fibrations over $\Lambda$. The fibre $W_{\text {loc }}^{\text {uu }}(\Lambda, p)$ at $p \in \Lambda$ is characterised by $W_{\text {loc }}^{\text {mu }}(\Lambda, p)=\{z$ $\in W_{\mathrm{loc}}^{u}(\Lambda): d\left(f^{n}(z), f^{n}(p)\right) \rightarrow 0$ as $\left.n \rightarrow-\infty\right\}$. Similarly for $W_{\mathrm{loc}}^{s s}(\Lambda, p)$.

(e) There exist an open neighbourhood $Q$ of $f$ in $\operatorname{Diff}_{G}^{r}(M)$, an invariant neighbourhood $V$ of $\Lambda$ and continuous maps $\Gamma, \Gamma^{u}, \Gamma^{s}: Q \rightarrow \operatorname{Diff}_{G}^{r}(M)$ such that

(i) $\Gamma(f)=\Gamma^{u}(f)=\Gamma^{s}(f)=$ identity map of $M$.

(ii) For $f \in Q, \Gamma\left(f^{\prime}\right)(\Lambda)=\Lambda^{\prime}$ is contained in $V$ and is an elementary periodic orbit of $f^{\prime}$ of the same period as $\Lambda$.

(iii) $\Gamma^{u}\left(f^{\prime}\right)\left(W_{\text {loc }}^{u u}(\Lambda, p)\right)=W_{\text {loc }}^{u u}\left(\Lambda^{\prime}, \Gamma\left(f^{\prime}\right)(p)\right), p \in \Lambda, f^{\prime} \in Q$. Similarly for $W^{u}$, $W^{s s}$ and $W^{s}$.

(f) (a)-(e) similarly hold for flows and $\Lambda$ an elementary (periodic) orbit.

Proof. Theorem $A$ is essentially a special case of Theorem 4.1 of [15]. We note that we can obtain a $C^{r}$ locally trivial fibration rather than just lamination in (d) because $\Lambda / G$ is finite (for flows, this follows from the transitivity of the induced flow on $\Lambda / G \cong S^{1}$ ). (e) is a consequence of part (f) of Theorem 4.1 of [15] together with the equivariant isotopy theorem ([6, Theorem 3.1, Chapter 6]).

Although (e)(ii) is a consequence of Theorem 4.1 of [15], we prefer to indicate an elementary proof avoiding stable manifold theory. Suppose $\Lambda \subset M_{i}$. If $\Lambda$ is elementary it consists of $p$ nondegenerate orbits for $f^{p}$ on $M_{i}, p=\operatorname{period}$ of $\Lambda$ (Definition A2, §3). Since nondegeneracy is framed in terms of a transversality condition we can then use the openness and stability of transversal intersections to deduce the persistence of the invariant set $\Lambda$ under perturbation. The continuity of spec will guarantee that the new invariant set is still elementary and Thom's isotopy theorem [1, p. 51] together with the equivariant isotopy theorem will give us the isotopy statements in (e)(ii).

We have the following equivariant versions of Hartman's theorem for elementary invariant and periodic orbits. We recall that $f$ and $g$ are said to be conjugate if there exists a homeomorphism $h$ such that $f g=h g$.

THEOREM B1. Let $\Lambda$ be an elementary periodic orbit for $f \in \operatorname{Diff}_{G}^{1}(M), T \Lambda \oplus N^{u}$ $\oplus N^{s}$ denote the corresponding splitting of $T_{\Lambda} M$ and $N f=T f \mid N^{u} \oplus N^{s}$. Then $f$ is conjugate to $N f$ near $\Lambda$ by an equivariant homeomorphism.

TheOREM B2. Let $X \in C_{G}^{1}(T M)$ and suppose $\Lambda$ is either an elementary G-orbit or an elementary periodic orbit for $X$. Then $\left\{N F_{t}^{X}\right\}$ is conjugate to $\left\{F_{t}^{X}\right\}$ near $\Lambda$ by an equivariant homeomorphism which is independent of $t$.

Proofs OF B1 AND B2. Theorem 4.1 of [15], [22].

Proposition C. If $X \in C_{G}^{1}(T M), \Lambda$ is an elementary periodic orbit of $X$ of period $T$ and $\mu>T$, there exists an open neighbourhood $Q$ of $X$ in $C_{G}^{1}(T M)$ and an invariant 
neighbourhood $U$ of $\Lambda$ in $M$ such that if $Y \in Q, Y$ has a unique periodic orbit $\Lambda_{Y}$ wholly contained in $U$. Furthermore, $\Lambda_{Y}$ is elementary, of period $<\mu$ and any periodic orbit of $Y$ meeting $U$ is of period $>\mu$.

A similar result holds for diffeomorphisms.

Proof. We prove this proposition for flows; the proof is similar for diffeomorphisms. By Theorem B2, we may find an invariant neighbourhood $W$ of $\Lambda$ such that $\left\{F_{t}^{X}\right\}$ is conjugate to $\left\{N F_{t}^{X}\right\}$ in $W$. Choosing $W$ smaller if necessary, we may therefore assume that $\Lambda$ is the unique orbit of period $\leqslant \mu$ meeting $W$. Working inside $W$, we choose $C^{1}$ equivariant fundamental domains $D_{u}$ and $S_{s}$ for $\left\{F_{t}^{X}\right\}$ contained in $W_{\text {loc }}^{u}(\Lambda)$ and $W_{\text {loc }}^{s}(\Lambda)$ respectively [22, p. 192]. Choose $r>0$ such that the closed disc bundle of radius $r$ of the normal bundle of $W_{\text {loc }}^{u}(\Lambda)$ restricted to $D_{u}$ embeds as a closed $G$-invariant submanifold $X_{u}^{r}$ of $W$ transverse to $W_{\text {loc }}^{u}(\Lambda)$. We similarly define $X_{s}^{t}$ as an embedded submanifold of $W$ transverse to $W_{\text {loc }}^{s}(\Lambda)$ along $D_{s}$. We shall suppose $r$ and $t$ are chosen sufficiently small so that $X$ is transverse to $X_{u}^{r}$ and $X_{s}^{t}$. Taking $t$ smaller if necessary, $F^{X}$ induces a $C^{1}$ map $\theta_{X}: \partial X_{s}^{t} \rightarrow X_{u}^{r / 2}$. Thus, $\theta_{X}(x)=F(x, \gamma(x))$, where $\gamma: \partial X_{s}^{t} \rightarrow R(>0)$ is $C^{1}$ and gives the first time of intersection of the forward orbit through $x$ with $X_{u}^{r}$. By Theorem B2, we may suppose $t$ is chosen so that if $x \in X_{s}^{t}$ then the forward orbit through $x$ is either asymptotic to $\Lambda$ or meets $X_{u}^{r / 2}$ after time at least $\mu+1$. By the $\lambda$-lemma [19], the collection of orbits through $X_{s}^{t}$, together with $W_{\text {loc }}^{u}(\Lambda)$, fill out a neighbourhood of $\Lambda$. Let $V(X)$ denote that part of the neighbourhood bounded by $X_{s}^{t}, X_{u}^{r}$ and the orbits $F_{t}(x), x \in \partial X_{s}^{t}, t \in[0, \gamma(x)]$. Fix an invariant open neighbourhood $U$ of $\Lambda$ such that $U \subset V(X)$ and $\bar{U} \cap \partial V(X)=\varnothing$. By Theorem A, we may find an open neighbourhood $Q$ of $X$ such that if $Y \in Q$, then $Y$ has a periodic orbit $\Lambda_{Y} \subset U$ of period $<\mu$ and $\Lambda_{Y}$ and the local stable and unstable manifolds of $Y$ depend continuously on $Y$ in the $C^{1}$ topology. For possible smaller $Q$ we may by the above and the parametrised flow theorem (Theorem 21.4 of [1]) require that if $Y \in Q$ then

1. $X_{s}^{t}, X_{u}^{r}$ are transversal to $Y$ and meet the local stable and unstable manifolds of $\Lambda_{Y}$ in fundamental domains.

2. $\theta_{Y}: \partial X_{s}^{t} \rightarrow X_{u}^{r}$.

3. $V(Y) \supset U$ and $\partial V(Y) \cap \bar{U}=\varnothing$.

4. If $x \in X_{s}^{t}$, then either $x$ is asymptotic to $\Lambda_{Y}$ or the forward $F^{Y}$-orbit through $x$ meets $X_{u}^{r}$ after time $\geqslant \mu$.

It now follows that if $x \in X_{s}^{t}$ lies on a periodic orbit of $Y$ then the orbit must have period $>\mu$. But every point in $U \subset V(Y)$ is either on an orbit through $X_{s}^{t}$ or on the local unstable manifold of $\Lambda_{Y}(\lambda$-lemma).

\section{Perturbation lemmas.}

LemmA A. Let $\alpha$ be an $X$-invariant $G$-orbit, $X \in C_{G}^{r}(T M)$, and $U$ be an invariant open neighbourhood of $\alpha$. Then there exists $Y \in C_{G}^{r}(T M)$ arbitrarily $C^{r}$ close to $X$ such that $\alpha$ is an elementary $G$-orbit for $Y$ and $Y=X$ on $M \backslash U$.

Similarly for diffeomorphisms. 
Proof. We prove for vector fields; the proof for diffeomorphisms is similar. As in $\S 1$, we let $q: N \rightarrow M$ denote an embedding of the normal bundle of $\alpha$ as a tubular neighbourhood of $\alpha$ contained in $U$. We may suppose that $q$ equals the exponential map on some neighbourhood of the zero section of $N$. For $\lambda \in \mathbf{R}$, we define an equivariant vector field $\theta_{\lambda}$ on $N$ by $\theta_{\lambda}(v)=\lambda v$ (multiplication in fibres). Set $Z_{\lambda}=q_{*}\left(\theta_{\lambda}\right)$. Using an equivariant bump function supported in $q(N)$, we construct $Y_{\lambda} \in C_{G}^{\infty}(T M)$ such that $Y_{\lambda}=Z_{\lambda}$ on some invariant neighbourhood of $\alpha$, equals zero outside $U$ and depends linearly on $\lambda$. Clearly as $\lambda \rightarrow 0, Y_{\lambda} \rightarrow 0, C^{r}$. Now Hess $\left(X+Y_{\lambda}\right)=\operatorname{Hess}(X)+\operatorname{Hess}\left(Y_{\lambda}\right)$. Since Hess $\left(Y_{\lambda}\right)=\lambda$ (the derivative of $\exp =q$ on the zero section is the identity), we may choose $\lambda$ arbitrarily close to zero so that $0 \notin \operatorname{Hess}\left(X+Y_{\lambda}\right)$. But then $Y=X+Y_{\lambda}$ will satisfy the conditions of the lemma.

LEMMA B. Let $X$ and $Y$ be compact $G$-manifolds with boundary, $f: X \rightarrow Y$ be a $C^{r}$ equivariant embedding such that $f(\partial X) \subset Y \backslash \partial Y$ and $0<a<b$. Then there exists an open neighbourhood $Q$ of $f$ in $C_{G}^{r}(X, Y)$ such that if $g \in Q$ then $g$ is an embedding and there exists a $C^{r}$ equivariant isotopy $H: X \times[a, b] \rightarrow Y$ between $f$ and $g$ satisfying

1. $H_{t}(x)=f(x), t \in[a, b]$, whenever $f(x)=g(x)$.

2. $H_{t}=f$ for $t$ close to $a ; H_{t}=g$ for $t$ close to $b$.

3. $H$ depends continuously on $g$ in $C^{r}$ topology.

4. The $t$-derivative of $H$ is $C^{r}$ in the $(x, t)$-variable.

Proof. The result is an equivariant version of a standard isotopy lemma and we only sketch the proof. Fix an equivariant Riemannian metric on $Y$. For $g$ close to $f$, there exists a unique geodesic $\gamma_{x}(g):[0,1] \rightarrow Y$ joining $f(x)$ and $g(x)$ and $\gamma$ depends $C^{r}$ on $x$ and continuously on $g, C^{r}$ topology. Define $H(x, t)=\gamma_{x}(g)(k(t))$, where $k:[0,1] \rightarrow[a, b]$ is $C^{\infty}$ and equal to $a$ (resp. $b$ ) on a neighbourhood of 0 (resp. 1 ).

Let $\Lambda$ be a periodic orbit of $X$ and suppose $\left(D, D^{\prime}, P, \rho\right)$ is a Poincaré system for $\Lambda$. We say that an open invariant neighbourhood $U$ of $\Lambda$ is subordinate to $\left(D, D^{\prime}, P, \rho\right)$ if $\bar{U} \subset \cup_{x \in D^{\prime}} F(x,[0, \rho(x)])$.

Lemma C. Let $\Lambda$ be a periodic orbit of $X \in C_{G}^{r}(T M),\left(D, D^{\prime}, P, \rho\right)$ be a Poincaré system for $\Lambda$ and $U$ be an open invariant neighbourhood of $\Lambda$ subordinate to $\left(D, D^{\prime}, P, \rho\right)$. We may find a tubular neighbourhood $V$ of $\Lambda$, an open neighbourhood $Q$ of $P$ in the subset of $C_{G}^{r}\left(D^{\prime}, D\right)$ consisting of maps equal to $P$ outside $V \cap D^{\prime}$ and a continuous map $\chi: Q \rightarrow C_{G}^{r}(T M)$ such that

1. $\chi\left(P^{\prime}\right)$ has Poincaré map $P^{\prime}, P^{\prime} \in Q$.

2. $\chi\left(P^{\prime}\right)=X$ on $M \backslash U, P^{\prime} \in Q$.

3. $\chi(P)=X$.

Proof. Choose $V$ so that $F(x,[0, \rho(x)]) \subset U$ for all $x \in D^{\prime} \cap \bar{V}$. Fix positive numbers $a$ and $b$ satisfying $0<a<b<\inf _{x \in D^{\prime}} \rho(x)$. Suppose $P^{\prime} \in C_{G}^{r}\left(D^{\prime}, D\right)$ equals $P$ outside $V \cap D^{\prime}$. For $P^{\prime}$ sufficiently $C^{r}$ close to $P$ we can, by Lemma $B$, find a $C^{r}$ equivariant isotopy $H: \bar{D}^{\prime} \times[a, b] \rightarrow D$ between the inclusion $i: \bar{D}^{i} \rightarrow D$ and $P^{-1} P^{\prime}$ (here we take $P^{-1} P^{\prime}=i$ on $\partial D^{\prime}$ which is permissible since $P=P^{\prime}$ on a neighbourhood of $\partial D^{\prime}$ in $\left.D^{\prime}\right)$. Set $\tilde{F}_{y}(t)=F\left(H_{t}(y), t\right), y \in D^{\prime}, t \in[0, \rho(y)]$. 
$\tilde{F}_{y}(\rho(y))=F\left(P^{-1}\left(P^{\prime}(y)\right), \rho(y)\right)=P^{\prime}(y)$, by definition of $P$ and $\rho, \tilde{F}_{y}(t)=F_{y}(t)$ for $y$ in some neighbourhood of $\partial D^{\prime}$ in $D^{\prime}$. By condition 3 of Lemma $\mathrm{B}$, if $y \in V \cap D^{\prime}$, none of the curves $\tilde{F}_{y}(t)$ will meet $M \backslash U$ provided $P^{\prime}$ is sufficiently $C^{r}$ close to $P$. By the openness of embeddings, it follows that if $P^{\prime}$ is sufficiently $C^{r}$ close to $P$, then $\tilde{F} \mid D^{\prime} \times[a, b]$ is an embedding and hence the curves $\tilde{F}_{y}$ are mutually disjoint. We define a vector field $X^{\prime}$ on the image of $\tilde{F}$ by setting $X^{\prime}\left(\tilde{F}_{y}(t)\right)=\tilde{F}_{y}^{\prime}(t) . X^{\prime}$ is $C^{r}$ and well defined. Since $X^{\prime}=X$ outside $U, X^{\prime}$ extends $C^{r}$ to $M$ if we set $X^{\prime}=X$ on $M \backslash U$. The integral curves of $X^{\prime}$ are given, as sets, by $\tilde{F}_{y}$. Thus the Poincare map for $X^{\prime}$ equals $P^{\prime}$. The correspondence $P^{\prime} \rightarrow X^{\prime}$ gives the map $\chi$ in the statement of the lemma. The continuity of $\chi$ follows from condition 3 of Lemma $B$.

REMARK. Lemma $\mathrm{C}$ has the following converse (proved by using the parametrised flow theorem). Let $\left(D, D^{\prime}, P, \rho\right)$ be a Poincaré system for the periodic orbit $\Lambda$ of $X \in C_{G}^{r}(T M)$. Suppose $\overline{P\left(D^{\prime}\right)} \subset D$. Then we may find an open neighbourhood $Q$ of $X$ in $C_{G}^{r}(T M)$ such that if $Y \in Q$ then the Poincaré map $P_{Y}$ : $D^{\prime} \rightarrow D$ for $Y$ is defined and $P_{Y}$ depends continuously on $Y, C^{r}$ topology (see also Proposition $\mathrm{C}$ of $\$ 5$ ).

\section{Density theorems. I.}

Definition A. A vector field $X \in C_{G}^{r}(T M)$ is said to be 1-generic if every $X$-invariant $G$-orbit is elementary.

Similarly for diffeomorphisms.

Definition B. A vector field $X \in C_{G}^{r}(T M)$ is said to be 2-generic if it is 1 -generic and every $X$-invariant periodic orbit is elementary.

Similarly for diffeomorphisms.

Recall that a subset of a topological space is said to be residual if it is the intersection of a countable family of open and dense sets. A residual subset of a complete metric space is, by the Baire category theorem, dense.

The aim of this section is to prove

TheOREM C. Let $M$ be a compact $G$-manifold. Then

1. The set of 1-generic $C^{r}$ vector fields is an open and dense subset of $C_{G}^{r}(T M)$, $1<r<\infty$. Similarly for diffeomorphisms.

2. The set of 2-generic vector fields is a residual subset of $C_{G}^{r}(T M), 1<r<\infty$. Similarly for diffeomorphisms.

We shall present a proof of Theorem $C$ for vector fields. Our approach is similar to Peixoto's proof of the Kupka-Smale theorem [20]. The proof for diffeomorphisms follows that given for (nonequivariant) diffeomorphisms by Smale in [25].

LEMMA D. Let $K$ be a compact invariant subset of $M$. Then $\Gamma(K, T)=\{X \in$ $C_{G}^{r}(T M): K$ does not contain any $X$-invariant $G$-orbit and does not meet any periodic orbit of $X$ of period $\leqslant T\}$ is an open subset of $C_{G}^{r}(T M)$.

Proof. This lemma is an equivariant version of Lemma 3 in [20]. The proof depends on a compactness argument similar to that given by Peixoto and we omit details. 
As a corollary of the proof of Lemma D we have

LEMMA E. Let $K$ be a compact invariant subset of $M, T \geqslant 0$ and $X \in \Gamma(K, T)$. Then there exist an open invariant neighbourhood $W$ of $K$ and neighbourhood $Q$ of $X$ such that $Q \subset \Gamma(W, T)$.

LEMMA F. The set of 1-generic vector fields in $C_{G}^{r}(T M)$ is open.

Proof. Let $X$ be 1-generic and have invariant elementary $G$-orbits $\alpha_{1}, \ldots, \alpha_{k}$. By Proposition $\mathrm{C}$ of $\$ 5$, we may find an open neighbourhood $Q$ of $X$ and invariant open neighbourhoods $U_{i}$ of $\alpha_{i}$ such that if $Y \in Q$ then $Y$ has precisely one invariant $G$-orbit $\alpha_{i}(Y)$ in $U_{i}$ which is elementary, $1 \leqslant i \leqslant k$. We now apply Lemma D with $T=0$ and $K=M \backslash \cup \cup_{i=1}^{k} U_{i}$ to find open neighbourhood $Q \cap$ $\Gamma(K, 0)$ of $X$ consisting of 1-generic vector fields.

LEMMA G. The set of 1-generic vector fields in $C_{G}^{r}(T M)$ is dense.

Proof. Let $M^{j}=\cup_{i=1}^{j} M_{i}$, where the $M_{i}$ are as in $\S 1 . M^{j}$ is a compact invariant subset of $M$ and $M^{1}=M_{1}, M^{N}=M$. Let $\Gamma^{j}$ denote the subset of $C_{G}^{r}(T M)$ consisting of vector fields all of whose invariant $G$-orbits in $M^{j}$ are elementary. We shall show that $\Gamma^{j}$ is open and dense in $C_{G}^{r}(T M), 1<j<N$. Openness follows as in Lemma F, using Lemma D. For density we proceed by induction on $j$. We let $\Delta^{j}$ denote the subset of $C_{G}^{r}(T M)$ consisting of vector fields all of whose invariant $G$-orbits in $M^{j}$ are nondegenerate (\$3). Suppose $j=1$. Then $\Delta^{1}$ is certainly open and using Lemma B1 of $\$ 3$ and Thom's transversality density theorem $\left[1\right.$, p. 48] it is easy to verify that $\Delta^{1}$ is dense in $C_{G}^{r}(T M)$. The transversality conditions together with the compactness of $M_{1}$ guarantees that if $X \in \Delta^{\prime}$, then $X$ has only finitely many invariant $G$-orbits in $M_{1}$ which depend continuously on $X$. We now apply Lemma $A$ of $\S 6$, to find $X^{i} \in \Gamma^{1}$ arbitrarily $C^{r}$ close to $X \in \Delta^{1}$. Hence $\Gamma^{1}$ is dense in $C_{G}^{r}(T M)$. Now suppose $N>j>1$ and that $X \in \Gamma^{j}$. It follows from Lemma $E$ and Theorem $A$ of $\$ 5$ that there exist an open neighbourhood $Q$ of $X$ in $\Gamma^{j}$ and an open invariant neighbourhood $W$ of $M^{j}$ such that if $Y \in Q$ every invariant $G$-orbit of $Y$ in $W$ lies in $M^{j}$ and is elementary. Let $V$ be a closed invariant neighbourhood of $M^{j}$ contained in $W$. As for $j=1$, we may find $X^{\prime} \in \Delta^{j+1} \cap Q$ arbitrarily $C^{r}$ close to $X$ and equal to $X$ on $V$. Since $M^{j+1} \backslash W$ is compact, there are only finitely many $X^{\prime}$-invariant $G$-orbits in $M^{j+1}$ and so, using Lemma $A$ of $\S 6$, we may $C^{r}$ approximate $X^{\prime}$ by $X^{\prime \prime} \in \Gamma^{j+1}$. This argument completes the inductive step.

Lemmas $\mathrm{F}$ and $\mathrm{G}$ imply part 1 of Theorem $\mathrm{C}$.

For $T>0$, we let $B(M, T)$ denote the subset of $C_{G}^{r}(T M)$ consisting of 1-generic vector fields all of whose orbits of period $\leqslant T$ are elementary.

LemMa H. For $T \geqslant 0, B(M, T)$ is an open and dense subset of $C_{G}^{r}(T M)$.

Proof. The proof of this lemma is very similar to that of Proposition 1 in [20] and we only indicate the main points. Openness of $B(M, T)$ follows from Lemma $\mathrm{D}$ and Proposition $\mathrm{C}$ of $\S 5$. For density, we first show that if $X$ is 1-generic then there exists $\tau>0$ such that every periodic orbit of $X$ has period $>\tau$. Let $B^{j}(M, T)$ 
denote the subset of $C_{G}^{r}(T M)$ consisting of 1-generic vector fields all of whose orbits in $M^{j}$ of period $\leqslant T$ are elementary. From Proposition $\mathrm{C}$ of $\$ 5$ we see that if $X \in B^{j}(M, T)$ then $X$ has only finitely many periodic orbits in $M^{j}$ of period $<T$. The proof is completed by showing that $B^{j}(M, T)$ is open and dense, $1<j<N$. This is accomplished by induction on $j$, as in the proof of Lemma $\mathrm{F}$, together with Peixoto's inductive argument based on Proposition C of $\$ 5$, Lemmas A and C of $\$ 6$ and Lemma $E$ of the present section.

Since the set of 2-generic vector fields on $M$ equals $\bigcap_{n=0}^{\infty} B(M, n)$, the proof of Theorem $\mathbf{C}$ is complete.

8. Stable manifold theory. In this section we construct the global stable and unstable manifolds of elementary critical elements of equivariant vector fields and diffeomorphisms. As the theory is similar for vector fields and diffeomorphisms we present details only for the former.

Suppose that $\Gamma$ is an (elementary) critical element of $X \in C_{G}^{r}(T M)$ (that is, an $X$-invariant $G$-orbit or periodic orbit). We set

$$
\begin{aligned}
& W^{u}(\Lambda ; X)=\left\{z \in M: F_{t}^{X}(z) \rightarrow \Lambda, t \rightarrow-\infty\right\}, \\
& W^{s}(\Lambda ; X)=\left\{z \in M: F_{t}^{X}(z) \rightarrow \Lambda, t \rightarrow+\infty\right\} .
\end{aligned}
$$

We often abbreviate the stable and unstable manifolds of $\Lambda$ to $W^{s}(\Lambda)$ and $W^{u}(\Lambda)$ respectively when it is not necessary to exhibit the explicit dependence on $X$. For $p \in \Lambda$, we set

$$
W^{u u}(\Lambda, p ; X)=\left\{z \in M: d\left(F_{t}(z), F_{t}(p)\right) \rightarrow 0, t \rightarrow-\infty\right\}
$$

similarly for $W^{s s}(\Lambda, p ; X)$.

If $\Lambda$ is elementary, we have a $C^{r}$ splitting $T \Lambda \oplus N^{u} \oplus N^{s}$ of $T_{\Lambda} M$ as $C^{r}$ $G$-vector bundles over $\Lambda$.

TheOrem A. $W^{u}(\Lambda, X)$ is the image of a $C^{r}$ injective equivariant immersion $I^{u}(X): N^{u} \rightarrow M . I^{u}(X)$ maps the zero section of $N^{u}$ onto $\Lambda$ and $I^{u}(X)\left(N_{p}^{u}\right)=$ $W^{u u}(\Lambda, p ; X), p \in \Lambda$.

Similarly for $W^{s}(\Lambda, X)$.

Proof. It follows from the proof of Theorem 4.1 in [15], that $W_{\text {loc }}^{u}(\Lambda)$ (see §5) may be represented as the image of a $C^{r}$ equivariant embedding $I_{\text {loc }}^{u}(X): N^{u}(r) \rightarrow$ $M$, where $N^{u}(r)$ is the open disc bundle of radius $r>0$, and that $I_{\text {loc }}^{u}(X)\left(N_{p}^{u}(r)\right)=$ $W_{\text {loc }}^{u u}(\Lambda, p), p \in \Lambda$. Now clearly, $W^{u}(\Lambda)=\cup_{t<0} F_{t}\left(W_{\text {loc }}^{u}(\Lambda)\right)$ and we construct $I^{u}(X)$ using the standard "continuation" argument as detailed, for example, in [1, pp. 87-89]. A corollary of this construction is that we may require $I^{u}(X)\left(N_{p}^{u}\right)=$ $W^{u u}(\Lambda, p: X), p \in \Lambda$.

If $E$ is the total space of a $C^{r}$ vector bundle isomorphic to $N^{u}$ (not necessarily the same base) and $\rho^{u}: E \rightarrow M$ is a $C^{r}$ injective equivariant immersion onto $W^{u}(\Lambda)$ satisfying the conditions of Theorem $A$, we say that $\rho^{u}$ is a $C^{r}$ parametrisation of $W^{u}(\Lambda)$. Similarly for $C^{r}$ parametrisations of $W^{s}(\Lambda)$. 
THEOREM B. Let $\Lambda$ be an elementary critical element of $X \in C_{G}^{r}(T M)$ and $T \Lambda \oplus N^{u} \oplus N^{s}$ denote the corresponding splitting of $T_{\Lambda} M$. Then there exist an open neighbourhood $Q$ of $X$ in $C_{G}^{r}(T M)$, open invariant neighbourhood $V$ of $\Lambda$, continuous map $\Gamma: Q \rightarrow \operatorname{Diff}_{G}^{r}(M)$ and continuous maps $\rho^{u}: Q \rightarrow C_{G}^{r}\left(N^{u}, M\right), \rho^{s}: Q \rightarrow$ $C_{G}^{r}\left(N^{s}, M\right)$ such that

1. $\Gamma(Y)(\Lambda)=\Lambda_{Y}$ is the unique (elementary) critical element of $Y$ contained in $V$.

2. $\Gamma(X)(\Lambda)=\Lambda$.

3. $\rho^{u}(Y): N^{u} \rightarrow M$ is a $C^{r}$ parametrisation of $W^{u}\left(\Lambda_{Y}, Y\right)$. Similarly for $\rho^{s}(Y)$.

(We take the compact $C^{r}$ topology on $C_{G}^{r}\left(N^{s}, M\right)[14$, p. 34].)

Proof. The result follows straightforwardly from part (e) of Theorem A, §5, using the continuation argument of the proof of Theorem $A$ of the present section and we omit details (see also [1, Theorem 27.4]).

9. Density theorems. II. All maps in this section will be $C^{\infty}$ unless the contrary is specifically indicated.

In [4], [5], [9], [10] a theory of general position for equivariant maps is described. We shall follow the terminology of [9], [10] except that by " $G$-transversal" we shall mean "in equivariant general position" [5, p. 149] and not "zeroth approximation to equivariant general position". Given $f: M \rightarrow N$ and an invariant submanifold $W$ of $N$, the notation $f \pitchfork_{G} W$ will signify that $f$ is $G$-transversal to $W$. If $\alpha: X \rightarrow M, \beta$ : $Y \rightarrow M$ are injective equivariant immersions, $\alpha(X) \pitchfork_{G} \beta(Y)$ signifies that $\alpha \pitchfork_{G} \beta(Y)$ or, equivalently, that $\beta \pitchfork_{G} \alpha(X)$. It is in this sense that we shall talk about the $G$-transversality of (immersed) submanifolds.

Definition A. A vector field $X \in C_{G}^{\infty}(T M)$ is said to be 3-generic if it is 2-generic and the stable and unstable manifolds of the elementary critical elements of $X$ are $G$-transversal.

Similarly for diffeomorphisms.

We have the following generalisation of the Kupka-Smale density theorem.

TheOREM B. Let $M$ be a compact $G$-manifold. The 3-generic vector fields form a residual subset of $C_{G}^{\infty}(T M)$.

Similarly for diffeomorphisms.

Proof. Our proof is essentially that given by Peixoto in [20] and we only sketch the main details (the proof for diffeomorphisms follows Smale [25]). If $\Lambda$ is an elementary critical element of $X \in C_{G}^{\infty}(T M)$, we let $B^{s}(\Lambda)$ denote the image of the closed unit disc bundle of $N^{s}$ by a $C^{\infty}$ parametrisation of $W^{s}(\Lambda, X)$ (see $\S 8$ ) and set $B^{s}((\Lambda) ; t)=F_{t}^{X}\left(B^{s}(\Lambda)\right)$. Similarly for $B^{u}(\Lambda)$ and $B^{u}(\Lambda ; t)$.

Fix $X \in B(M, T)$ (see $\S 8)$ and let $\Lambda_{1}, \ldots, \Lambda_{q}$ denote the critical elements of $X$ of period $<T$. By Proposition $\mathrm{C}$, §5, we may find open neighbourhoods $U_{i}$ of $\Lambda_{i}$ and $Q_{i}$ of $X$ in $B(M, T)$ such that for $Y \in Q_{i}, Y$ has a unique elementary critical element $\Lambda_{i}(Y) \subset U_{i}$. Set $Q_{i j}=Q_{i} \cap Q_{j}$. As shown in [20], to prove the theorem it is sufficient to show that the set of vector fields $Y \in Q_{i j}$ for which $B^{s}\left(\Lambda_{i}(Y),-T\right) \pitchfork_{G} B^{u}\left(\Lambda_{j}(Y) ; T\right)$ is open and dense in $Q_{i j}$. Openness is a consequence of the openness of $G$-transversal intersections [5, p. 151] and Theorem B, 
§8. For density, fix $Y \in Q_{i j}$. Construct fences $\Sigma^{u}, \Sigma^{s}$ for the stable and unstable manifolds of $Y$ as in [20] (see also [22] and the proof of Proposition C, §5). By Theorem B of $\S 8$, we may find $\nu \gg 0$, a neighbourhood $Q^{\prime}$ of $Y$ and choose $\Sigma^{u}$ so small that $B^{u}\left(\Lambda_{i}(Z),-\nu\right) \cap B^{s}\left(\Lambda_{j}(Z),-T\right)=\varnothing$ and $F_{-\nu}^{Z}\left(\Sigma^{u}\right) \cap B^{s}\left(\Lambda_{j}(Z),-T\right)=$ $\varnothing, Z \in Q^{\prime}$. Choosing $\Sigma^{u}$ smaller if necessary, we may require that the map $\lambda$ : $\Sigma^{u} \times[-\nu, T] \rightarrow M ; \quad(x, t) \mapsto F_{t}^{Y}(x)$ is an embedding. Let

$$
C=F_{-T}^{Y}\left(\Sigma^{u} \cap B^{s}\left(\Lambda_{j}(Y) ;-T\right)\right) \text {. }
$$

Then $B^{u}\left(\Lambda_{i}(Y) ; T\right) \pitchfork_{G} B^{s}\left(\Lambda_{j}(Y),-T\right)$ if and only if $C \pitchfork_{G}\left(\partial B^{u}\left(\Lambda_{i}(Y)\right)\right)$. By the transversality density theorem for equivariant maps $[5$, p. 151] and the equivariant isotopy theorem [6], [14, p. 180] we may find an equivariant isotopy $\psi: \Sigma^{u} \times[0,1]$ $\rightarrow \Sigma^{u}$ which is arbitrarily $C^{\infty}$ close to the trivial isotopy and satisfies

1. $\psi_{0}=$ identity,

2. $\psi_{s}=$ identity on a collar of $\partial \Sigma$ in $\Sigma$.

3. $\psi_{1}\left(\partial B^{u}\left(\Lambda_{i}(Y)\right)\right) \pitchfork_{G} C$,

4. $\psi_{s}=\psi_{0}$ for $s$ close to 0 and $\psi_{s}=\psi_{1}$ for $s$ close to 1 .

Let $\theta: \Sigma^{u} \times[-\nu, T] \rightarrow \Sigma^{u} \times[-\nu, T]$ be the equivariant diffeomorphism defined by

$$
\begin{aligned}
\theta(x, t) & =(x, t), \quad-\nu \leqslant t<0, \\
& =(\psi(x, t / T), t), \quad 0 \leqslant t<T .
\end{aligned}
$$

Set $L=\lambda\left(\Sigma^{u} \times[0,1]\right)$ and let $E=(0,1)$ be the unit horizontal field on $\Sigma \times$ $[-\nu, T]$. We define

$$
\begin{aligned}
\bar{Y}(x) & =Y(x), \quad x \in M \backslash L, \\
& =T(\lambda \theta) E\left((\lambda \theta)^{-1}(x)\right), \quad x \in L .
\end{aligned}
$$

By the properties of $\psi$, we may require that $\bar{Y}$ is an equivariant vector field on $M$ which is $C^{\infty}$ close to $Y$ and, in particular, $\bar{Y} \in Q^{\prime}$. It follows easily from our construction of $\bar{Y}$ that $B^{s}\left(\Lambda_{i}(\bar{Y}),-T\right) \pitchfork_{G} B^{u}\left(\Lambda_{j}(\bar{Y}), T\right)$ (full details are given in [20]).

REMarks. 1. The local finiteness of $G$-orbit types together with Peixoto's argument in [20] show that Theorem B also holds on noncompact $G$-manifolds if we give $C_{G}^{\infty}(T M)$ the $C^{\infty}$ Whitney topology [13, p. 42].

2. At this time, there is no theory of general position for $C^{r}$ equivariant maps, $r<\infty$, and so we do not know whether a $C^{r}$ version of Theorem B holds. However, the openness statements in the proof of Theorem B are valid in the $C^{d}$-topology, where $d$ is finite and computed locally in terms of degrees of polynomial maps (see [10, pp. 434 and 446]).

10. Equivariant Morse-Smale systems. The $\Omega$-set of a diffeomorphism or flow is defined in [26]. From Pugh's closing lemma it follows that the set of diffeomorphisms $f$ which have periodic points dense in $\Omega(f)$ is residual in $\operatorname{Diff}^{1}(M)$ [26]. Easy examples show that this result is false for equivariant diffeomorphisms [12]. For this reason, we make a new definition of the $\Omega$-set of an equivariant diffeomorphism or flow. It was shown in [11] that given a compact $G$-manifold $M$, there exists a compact principal $G$-manifold $\hat{M}$ and equivariant map $\pi: \hat{M} \rightarrow M$ such 
that we have a continuous extension homomorphism $\operatorname{Diff}_{G}^{k+N-1}(M) \rightarrow \operatorname{Diff}_{G}^{k}(\hat{M})$; $\phi \mapsto \hat{\phi}$, where $k \geqslant 0$ and $N$ is the number of orbit types of $M$. $\hat{M}$ is called a resolution of $M$ and has the structure of a $G \times(\mathrm{Z} / 2)^{N-1}$ manifold.

Definition A. Let $f \in \operatorname{Diff}_{G}^{r}(M), r \geqslant N$. The (equivariant) $\Omega$-set of $f, \Omega_{G}(f)$, is defined to be $\pi(\Omega(\hat{f}))$, where $\hat{f}: \hat{M} \rightarrow \hat{M}$ is as described above.

We similarly define $\Omega_{G}$ for flows.

$\Omega(\hat{f})$ is a compact $G$-invariant subset of $\hat{M}$ and so $\Omega_{G}(f)$ is a compact $G$ - and $f$-invariant subset of $M . \Omega_{G}(f) \subset \Omega(f)$ and in general we do not have equality [12]. However, $\Omega_{G}(f)$ does contain the $\alpha$ - and $\omega$-limit sets of every point $x \in M$ [12].

Definition B. Let $f \in \operatorname{Diff}_{G}^{\infty}(M)$. We say $f$ is an equivariant Morse-Smale diffeomorphism if

1. $\Omega_{G}(f)$ consists of a finite set of elementary critical elements.

2. $f$ is 3-generic.

We similarly define equivariant Morse-Smale vector fields.

REMARK. We say an equivariant diffeomorphism or vector field is strongly equivariantly Morse-Smale if it satisfies the conditions of Definition B with $\Omega$ replacing $\Omega_{G}$.

Definition C. Let $f \in \operatorname{Diff}_{G}^{\infty}(M)$. We say $f$ is $G$-structurally stable if we can find a neighbourhood $U$ of $f$ such that for all $g \in u$ there exist an equivariant homeomorphism $h$ of $M$ and continuous map $Q: M \rightarrow G$ such that

(a) $Q(f(x)) h(f(x))=g h(x)$, for all $x \in M$.

(b) $Q \cdot h: M \rightarrow M$ is an equivariant homeomorphism.

Remarks. 1. We have to introduce $Q$ because we cannot control perturbations along invariant $G$-orbits.

2. We can strengthen Definition $\mathrm{C}$ by requiring openness in the $C^{d}$-topology, $d<\infty$. See $\$ 9$.

3. We may similarly define $G$-structural stability for equivariant vector fields.

We conjecture that equivariant Morse-Smale diffeomorphisms and vector fields are $G$-structurally stable.

We conclude this section by proving the following fundamental existence theorem.

THEOREM D. Every compact G-manifold admits a strongly equivariant MorseSmale vector field.

Proof. By a theorem of A. Wassermann [28, p. 150], there exists an equivariant Morse map $f$ on $M$, with critical locus a finite union of nondegenerate critical $G$-orbits. Fixing an equivariant Riemannian metric on $M$, we see that $\operatorname{grad}(f)$ is a 2-generic vector field $X$ on $M$ with no periodic orbits. (Notice that $X$ is zero restricted to its invariant $G$-orbits.) It remains to be proved that we may perturb $X$ to $X^{\prime}$ so that the stable and unstable manifolds of $X^{\prime}$ are $G$-transversal and no new invariant sets are introduced. Our proof now follows that of Smale in [24].

Pick mutually disjoint invariant open neighbourhoods $U_{1}, \ldots, U_{q}$ of the invariant $G$-orbits $\alpha_{1}, \ldots, \alpha_{q}$ of $X$. Let

$$
Q_{X}=\left\{Y: C_{G}^{\infty}(T M): Y=X \text { on } U_{1} \cup \cdots \cup U_{q}\right\} \text {. }
$$


Using the method of proof of Theorem B of $\$ 9$, we approximate $X$ by $X^{\prime} \in Q_{X}$ so that $W^{u}\left(X^{\prime}, \alpha_{j}\right) \pitchfork_{G} W^{s}\left(X^{\prime}, \alpha_{i}\right), 1 \leqslant i, j \leqslant q$. We may assume that no new critical elements are introduced since $X$ is transversal to the level surfaces of $f$ outside $U_{1} \cup \cdots \cup U_{q}$. Hence $X^{\prime}$ is strongly equivariantly Morse-Smale.

REMARK. We may choose an equivariant metric on $M$ such that $X^{\prime}=\operatorname{grad}(f)$ with respect to the new metric. See [24].

\section{REFERENCES}

1. R. Abraham and J. Robbin, Transversal mappings and flows, Benjamin, New York, 1967. MR 39 \#2181.

2. R. Abraham and J. Marsden, Foundations of mechanics, Benjamin, New York, 1971.

3. J. F. Adams, Lectures on Lie groups, Benjamin, New York, 1969.

4. E. Bierstone, General position of equivariant maps, Trans. Amer. Math. Soc. 234 (1977), 447-466.

5. __ Generic equivariant maps, (Nordic Summer School/NAVF, Sympos. in Math., Oslo, August 5-25, 1976), Sijthoff and Noordhoff, pp. 127-161.

6. G. E. Bredon, Introduction to compact transformation groups, Academic Press, New York, 1972.

7. M. J. Field, Equivariant dynamical systems, Bull. Amer. Math. Soc. 76 (1970), 1313-1318. MR 43 \# 3583.

8. __ Singularity theory and equivariant dynamical systems, Astérisque 40 (1976), 67-68.

9. __ Stratifications of equivariant varieties, Bull. Austral. Math. Soc. 16 (1977), 279-295.

10. __ Transversality in G-manifolds, Trans. Amer. Math. Soc. 231 (1977), 429-450.

11. __ Resolving actions of compact Lie groups, Bull. Austral. Math. Soc. 18 (1978), 243-254.

12. H_ Hyperbolicity for equivariant dynamical systems (to appear).

13. M. Golubitsky and V. Guillemin, Stable mappings and their singularities, Springer-Verlag, Berlin and New York, 1973. MR 49 \#6269.

14. M. W. Hirsch, Differential topology, Springer-Verlag, Berlin and New York, 1976.

15. M. W. Hirsch, C. C. Pugh and M. Shub, Invariant manifolds, Lecture Notes in Math., vol. 583, Springer-Verlag, Berlin and New York, 1977.

16. S. Kobayashi and K. Nomizu, Foundations of differential geometry, Vol. 1, Interscience, New York, 1963.

17. J. Marsden and M. McCracken, The Hopf bifunction, Springer Notes in Applied Math., SpringerVerlag, New York, 1976.

18. R. Palais, Foundations of global non-linear analysis, Benjamin, New York, 1968.

19. J. Palis, On Morse-Smale dynamical systems, Topology 8 (4) (1969), 385-405.

20. M. M. Peixoto, On an approximation theorem of Kupka and Smale, J. Differential Equations 3 (1966), 214-227.

21. L. S. Pontryagin, Topological groups, Princeton Univ. Press, Princeton, N. J., 1946.

22. C. C. Pugh and M. Shub, Linearization of normally hyperbolic diffeomorphisms and flows, Invent. Math. 10 (1970), 187-198.

23. M. Shub, Structurally stable diffeomorphisms are dense, Bull. Amer. Math. Soc. 78 (1972), 817-818.

24. S. Smale, On gradient dynamical systems, Ann. of Math. (2) 74 (1961), 199-206.

25. __ Stable manifolds for differential equations and diffeomorphisms, Ann. Scuola Norm. Sup. Pisa Cl. Sci. 18 (1963), 97-116.

26. __ Differentiable dynamical systems, Bull. Amer. Math. Soc. 73 (1967), 747-817.

27. Stability and isotopy in discrete dynamical systems, Dynamical Systems, M. M. Peixoto, Ed., Academic Press, New York, 1973, pp. 527-530.

28. A. G. Wassermann, Equivariant differential topology, Topology 8 (1969), 127-150. MR 40 \#3563.

Department of Pure Mathematics, University of Sydney, Sydney, N. S. W. 2006, Australia 